\title{
MOBILE APPLICATION TECHNOLOGY IN LEVELLING
}

\author{
Michal BEDNARCZYK ${ }^{1)}$ * and Artur JANOWSKI ${ }^{2)}$ \\ 1) Department of Land Surveying and Geomatics, Faculty of Geodesy and Land Management, University of Warmia and \\ Mazury in Olsztyn, ul. Heweliusza 12 (bl. 52), 10-724 Olsztyn, Poland \\ ${ }^{2)}$ Institute of Geodesy, Faculty of Geodesy and Land Management, University of Warmia and Mazury in Olsztyn, \\ ul. Oczapowskiego 1, 10-719 Olsztyn, Poland
}

*Corresponding author's e-mail: michal.bednarczyk@uwm.edu.pl

\begin{tabular}{l} 
ARTICLE INFO \\
\hline Article history: \\
Received 23 September 2013 \\
Accepted 7 March 2014 \\
Available online 27 March 2014 \\
\hline Keywords: \\
Mobile application \\
Levelling \\
Teleprocessing \\
Android OS
\end{tabular}

\section{ABSTRACT}

The topic of this article is the use of mobile application technology in geodetic measurements with an emphasis on levelling. Reference points were registered as data from levelling benchmarks, performed with a traditional dumpy level. The created application allowed the recording of measurements on location, sending them to a remote server for processing and preparing a report to be saved in a database. The project was to decrease the labor-intensive geodesy work and speed up the processing of measurement results and also to allow immediate access for all interested and authorized recipients of the results.

\section{INTRODUCTION}

Users of contemporary software can choose from an increasing number of a rich and constantly developing array of emerging applications. Even though methods for geodesic calculations and measurements were devised over one hundred years ago and computer programs which perform these algorithms were created mostly at the end of the 20th century, informatisation in the field continues to be a problem (Karney, 2013). Developmental trends of contemporary information technology solutions are their mobility, central data storage (Janowski et. al., 2006), as well as remote and distributed processing. This has been possible thanks to the huge and growing popularity of portable devices, above all the cellular telephone, smart phones and tab phones, which have become quite efficient miniature computers, equipped with a GPS module, electronic compass, digital camera, accelerometer, gyroscope and GSM/Wi-Fi communication.

Today this device is used for basically everything from standard services such as telephoning or text messaging to appointment planning, entertainment and GIS data processing and storage. Mobile technology is being used more widely in many areas including crisis management and environment monitoring, i.e. predicting floods or observing trembles in the Earth's crust in order to avert the results of tremors and earthquakes and monitor deformations in mines (Mirek, 2004; Blachowski et al., 2010, 2011). In addition, in GIS mobile applications mostly, mobile technology is being used in combination with augmented reality, which requires a new approach to user interface for cartographic presentation taking into account a interactive and dynamically changing perspective (egocentric view) (Halik, 2012). Thus, it is worth considering the possibilities of applying this modern technology in precise engineering tasks such as geodesic levelling.

Levelling are the operations connected with determining elevations or differences in elevations on Earth. There are various measurement methods including barometric, trigonometric and direct levelling, or with GNSS technology, each with its own faults and merits. This article concerns problems connected with direct levelling, which calculates differences in elevations between two points through targeting along a horizontal line (line of sight) to level staffs standing at those points. Segment readings are taken with the staffs showing the distance from the target line (backsight and foresight) (Kosiński, 2011; Łyszkowicz, 2010), and based on them, differences in elevation between points can be calculated.

Regardless of the equipment used - optical (dumpy) or digital, level measurements require further work, usually with computer programs. In the case of an optical level, gathered data has to be entered manually into the computer system (application). So far, there has been no solution for greater automation or remote gathering of level measurements using optical levels. In the opinion of the authors, the application of mobile technology could make levelling with optical levels significantly easier and more reliable.

The basic premise is that the current technology for geodesic levelling does not fully utilize mobile applications with remote access to centrally stored 


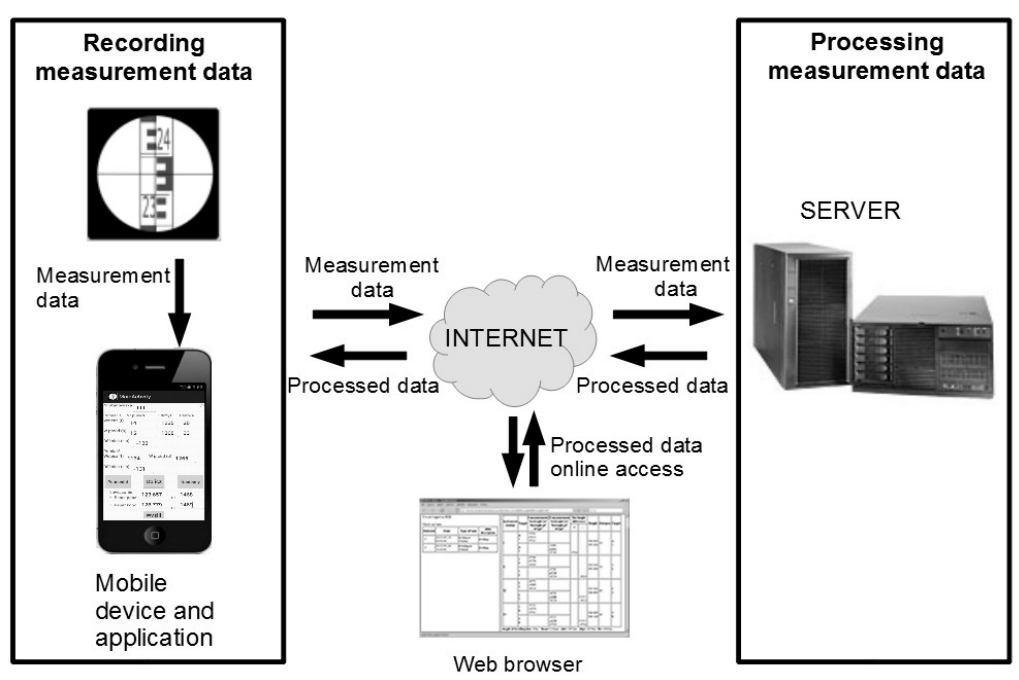

Fig. 1 Communication scheme. Source: Own work.

data (lack of such mobile geodesic solutions particularly for the increasingly popular Android open platform system). This concerns above all levelling with the use of a traditional dumpy level which lacks all the advanced electronic improvements of the tachometer or laser scanner and cannot automatically record measurements (except for level codes). With the use of mobile technology at the same time, these measurements could be done quickly, increasing the effectiveness of conducting and compiling measurements and lowering the costs of modern and productive equipment.

It was determined that the presented goal could be achieved through the acquisition of data with the use of mobile technology through the registration of data in electronic form during the measuring, controlling and recording in the central data repository with remote Internet access. The proposed solution was implemented in the form of a dedicated application in association with an Internet service. The authors could not find something similar among the known technologies, which was another essential premise for undertaking this project.

\section{MOBILE APPLICATIONS IN THE ANDROID SYSTEM}

A mobile application runs on a portable device like a tablet or cell phone and is determined by its network characteristics. It often uses the Internet to either gather or present data, which improves the quality of programming, and the user is not limited to the location of data and can receive updates, store data, manage and make accessible for others. An additional asset is the remote processing and execution of any operation from any point on Earth. Of course, Internet access is required for it be fully functional.

The Android System was created by the company Google with portable devices in mind. Many other platforms such as Symbian, BlackBerry OS, Windows Mobile and IPhone OS were all designed for the same purpose, but only Android is a completely open platform so it can be easily adapted to various devices. The creators of Android valued above all versatility, which is why it is not, unlike the competition, designated for any one appliance. This approach has resulted in Android becoming the more popular and dominant system.

Android is one of the many available operating systems dedicated to mobile solutions, but it is distinguished by three fundamental features (Conder et al., 2011):

1. Completeness. Designers and creators of the platform dealt with the problem extensively and comprehensively and devised a safe system as well as a framework for creating applications.

2. Openess. Android is made accessible based upon the principle of open source. Programmers have unparalled access to many different devices which use this system.

3. Free Access. The creators of the programming do not incur any actual costs or license fees from using the platform's services or writing programs. They do not have to sign even an application certification. Plus, there are many ways to obtain applications.

Taking into account these features and Android's growing popularity, the paper's authors decided to create this project based on this system.

\section{APPLICATION TO ASSIST IN LEVELLING MEASUREMENTS}

The heart of Android is the Linux System, but Java is the most commonly used programming language for creating applications. The authors used the free environment Eclipse ADT while writing the levelling application program and tested it on emulators (provided by Google platforms of Android 2.2 to 4.2) and on devices such as Motorola Defy with Android 2.2 (API level 8), Samsung Galaxy SI with Android 2.3.6 (API level 10) and Samsung Galaxy SII with Android 4.1.2 (API level 16). The proposed solution is composed of two elements (Fig. 1): 


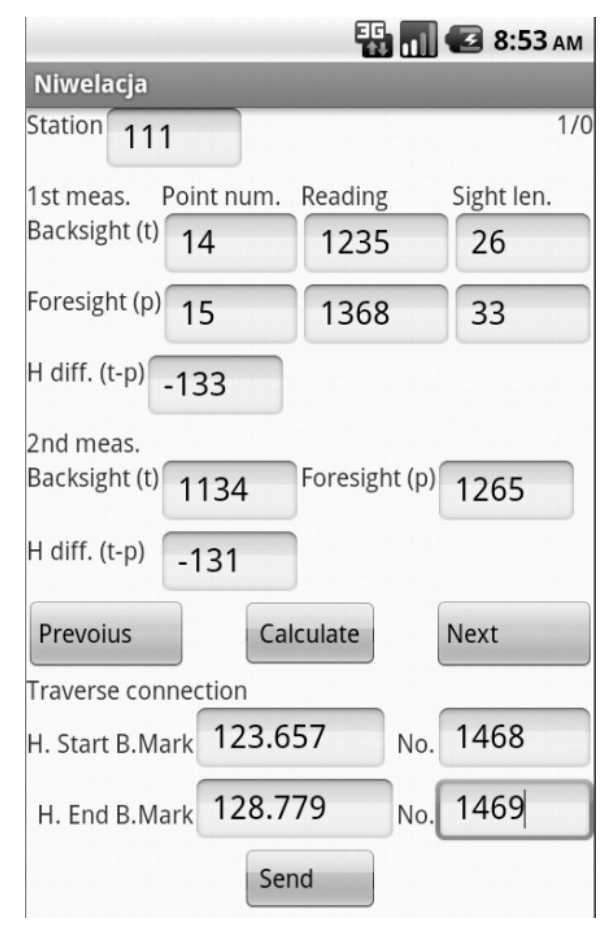

Fig. 2 Main screen of mobile application. Source: own work.

1. An application for recording measurements operating on a mobile device (smartphone, tabphone, tablet) with web access.

2. Internet service for processing measurement data.

Operating on a mobile device, the application makes it possible to record data during line levelling. Because this version of the application is used for entering measurements done with an optical levelling instrument, the user manually enters readings as if in a level book (Fig. 2). They enter:

- current number of level station,

- first "back" measurement (backsight - t):

- target/point number, staff reading, sight length (distance to staff),

- first "forward" measurement, (foresight - p):

- target/point number, staff reading, sight length,

- second "back" measurement:

- $\quad$ staff reading

- second "forward" measurement:

- $\quad$ staff reading

After the data is entered, the application calculates the height difference and initially verifies the result. The difference between calculated height differences of the first and second measurements must not exceed $3 \mathrm{~mm}$. If this occurs, the application informs that the measurement must be repeated. If all data are correct, the user presses the "Next" button to continue measurements at the next station. After this process, the user enters measurement results from the

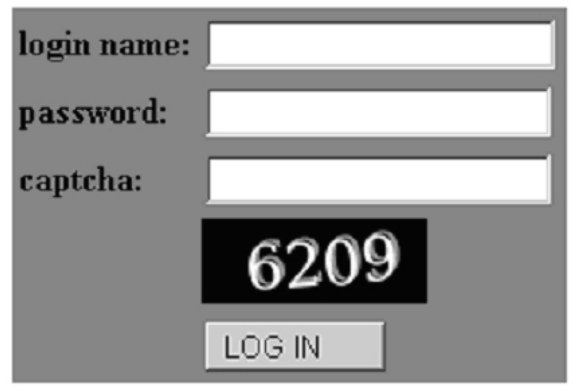

Fig. 3 User Login Panel. Source: Own work

other stations. All data is kept in the mobile device's memory, for when entries from a station or possible correction can be reviewed.

In the lower part of the screen, there is a space where the user enters numbers and elevations of reference points (initial and final benchmarks: H.Start B.Mark, H.End B.Mark). After the measuring is completed, the data is sent via the Internet to the servers, where sequences are aligned, calculated results are sent to the system customer (of the android application) and data is stored (in the server's data space).

Along with the mobile application, the user has at their disposal an online portal where the gathered data is processed. An authorized user (Fig. 3 - the window of an authorized user) can access archived data with the help of a standard Internet browser. The simplest way to view data is with a dynamically generated report (HTML format), presented as feedback (Fig. 4). In addition to visualizing exact calculations, the system transfers them to the relevant data formats used in the engineering tasks.

The server-side application is implemented in PHP scripting language, which was chosen especially for the availability of this technology in most network service providers (server providers). The server-side application is to allow users to access its calculation function (geodesy calculations) and input and make available gathered data. For integration, data are is stored on the MySQL server, with tables presented in Figure 5.

The suggested functional significance of the different database tables and their included columns is explained below. The "Levelling Tasks" table is the main table for storing direct levelling measurements (levelling benchmarks). The remaining tables are connected with reports for the purpose of preventing redundant data. Its construction is as follows:

ID - auto incremented field, main key,

STATION - name of measurement station

BACK_TARGET - name of back target

BBENC $\bar{C}$ HMARK - feature of back target ( if not null - pointed to the appropriate record of the benchmarks table)

FORWARD_TARGET - name of forward target FBENCHMARK - feature of back target ( if not null 


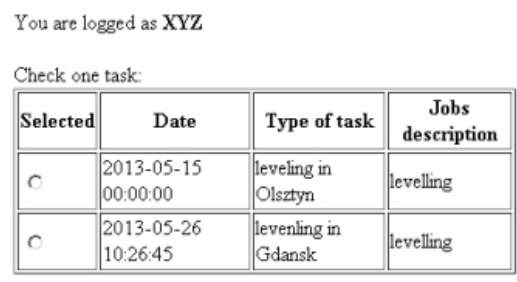

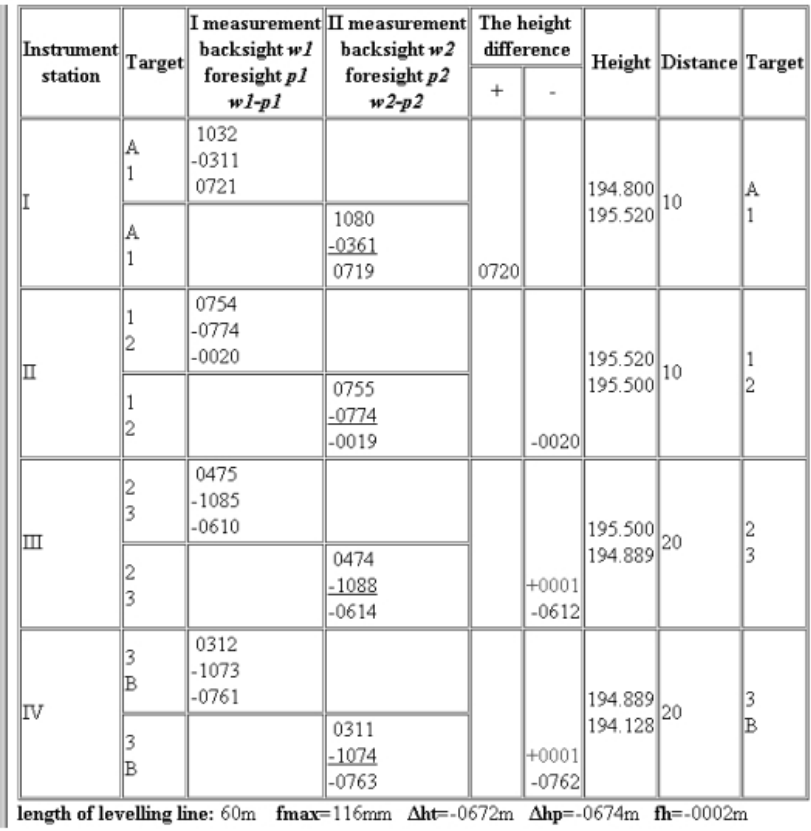

Fig. 4 HTML report.

Source: Own work.

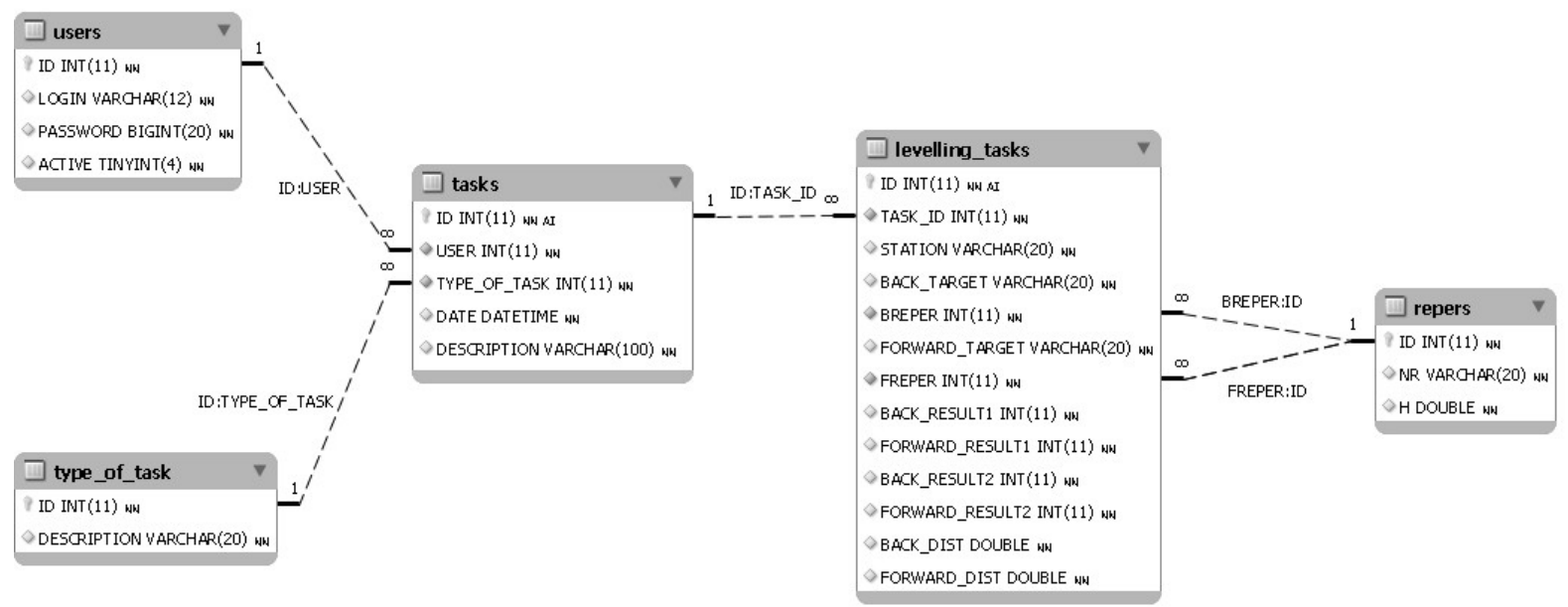

Fig. 5 Structure of the database. Source: Own work.

- pointed to the appropriate record of the benchmarks table)

BACK_RESULT1, BACK_RESULT2 - values of two back readings

FORWARD_RESULT1, FORWARD_RESULT2 values of two forward readings

BACK DIST, FORWARD DIST - measurement station distances from back and forward targets

The "Levelling Tasks" table is the primary chart of the system in which the processing of measurements is only one of the elements. The contents of its fields in some records determines the usage of the other charts logically related to different geodesy tasks. It contains the following columns:
ID - auto incremented field, main key,

USER - user identification pointing to the record in the users table

TYPE_OF_TASKS - type of task pointing to the record in the type_of_tasks table (the system will be further developed to add on other measurement tasks)

DATE - date of establishing task in system

DESCRIPTION - additional description facilitating identification of measurement task stored in the system

The "Type_of_Tasks" table is a standard table (vocabulary table) which arranges the stored data according to the type of geodesic task. Its structure is as follows: 
ID - auto incremented field, main key,

DESCRIPTION - description of measurement task (the system will be further developed to add on other measurement tasks)

(the system will be further developed to add on other measurement tasks

The "Users" table is for authorizing users and storing their assigned entitlements. The columns are:

ID - auto incremented field, main key,

LOGIN - name of user, used for their authorization in the system

PASSWORD - user's :hash password

ACTIVE - field for user's privileges (active, prohibited from using system, limited permission, etc.)

the "Benchmarks" table is the auxiliary table used for facilitating access to the information system about the recorded benchmark database

ID - auto incremented field, main key,

NR - Benchmark name

$\mathrm{H}$ - benchmark ordinate

The application is currently being tested in the field. The collected observations will serve to perfect the design and increase performance.

\section{SUMMARY AND CONCLUSIONS}

The aim of this work was to provide a solution for utilizing mobile application technology in levelling. Despite the incompatibility in equipment a lack of communication between a dumpy level and a smartphone and the necessity to enter measurements manually - the application of such technology seems to be well-founded as it allows for remote processing and sharing of data using optical levelling instrument and a mobile application. Such a task had been impossible to perform, but now the application of this method could speed up the computing of results because they are entered immediately into the user's registered account. Electronic data input replaces filling in a level book by hand with the digital version of current entries into a system's database.

The collected data as part of the user's account can be easily available which in turn allows field and office work to be done simultaneously without the measuring team having to return from the field. This solution plays a major role in conducting large measurement campaigns during which significant amounts of data are gathered and analyzed simultaneously. Four precise levelling campaigns have been conducted covering all of Poland, the last taking place between 1997 and 2003 (Kowalczyk et al., 2011). This project, after first being adapted to precise levelling, would greatly simplify the creation of a database for a measuring campaign.

The authors plan to improve the design by adding some services such as GPS localization. By doing this it will be possible to perform other tasks such as the automatic creation of a digital terrain model for geodynamical, geological or environmental exploration or research. In addition, there may be many uses of the proposed mobile technology. In the opinion of the authors, widespread availability of mobile applications will improve the quality of geodetic measurements.

\section{REFERENCES}

Blachowski, J., Ellefmo, S. and Ludvigsen, E.: 2011, Monitoring system observations of rock mass deformations caused by sublevel caving mining system. Acta Geodyn. Geomater., 8, No. 3 (163), 335344.

Blachowski, J., Milczarek, W. and Cacoń, S.: 2010, Project of a rock mass surface deformation monitoring system in the Walbrzych Coal Basin. Acta Geodyn. Geomater., 7, No. 3 (159), 349-354.

Conder, S. and Darcey, L.: 2011, Android Wireless Application Development, Second Edition, Pearson Education Inc.

Halik, Ł.: 2012, The analysis of visual variables for use in the cartographic design of point symbols for mobile augmented reality applications. Geodesy and Cartography 61 , No. 1, 19-30 DOI: $10.2478 / \mathrm{v} 10277-012-0019-4$

Janowski, A., Sawicki, P. and Szulwic, J.: 2006, Internet database for photogrammetric close range applications. Commission V Symposium „Image Engineering and Vision Metrology".

Karney, C.F.F.: 2013, Algorithms for geodesics. Journal of Geodesy $87,1,43-55$ DOI: $10.1007 / \mathrm{s} 00190-012-0578-\mathrm{z}$

Kosiński, W.: 2011, Geodesy. Wydawnictwo Naukowe PWN, Warszawa, (in Polish).

Kowalczyk, K,, Bednarczyk, M. and Kowalczyk, A.: 2011, Relational database of four precise levelling campaigns in Poland. 9th International Conference on Environmental Engineering (ICEE) vol 5, Vilnius Gediminas Technical University Press Technika.

Łyszkowicz, A. and Łyszkowicz, S.: 2010, Surveying. Oficyna Wydawnicza Politechniki Warszawskiej.

Mirek, J.: 2004, Controling of measurement process of strong ground motion using mobile technologies in SEJS-NET System. Acta Geodyn. Geomater., 1, No. 1 (133), 77-81. 